\title{
Analysis and Optimization of Dynamic Measurement Precision of Fiber Optic Gyroscope
}

\author{
Hui Li, Liyang Cui, Zhili Lin, and Chunxi Zhang \\ School of Instrument Science and Optoelectronics Engineering, Beihang University, Beijing 100191, China \\ Correspondence should be addressed to Hui Li; lihui@buaa.edu.cn
}

Received 26 August 2013; Accepted 16 November 2013

Academic Editor: Bo-Chao Zheng

Copyright ( 2013 Hui Li et al. This is an open access article distributed under the Creative Commons Attribution License, which permits unrestricted use, distribution, and reproduction in any medium, provided the original work is properly cited.

\begin{abstract}
In order to improve the dynamic performance of high precision interferometer fiber optic gyroscope (IFOG), the influencing factors of the fast response characteristics are analyzed based on a proposed assistant design setup, and a high dynamic detection method is proposed to suppress the adverse effects of the key influencing factors. The assistant design platform is built by using the virtual instrument technology for IFOG, which can monitor the closed-loop state variables in real time for analyzing the influence of both the optical components and detection circuit on the dynamic performance of IFOG. The analysis results indicate that nonlinearity of optical Sagnac effect, optical parameter uncertainty, dynamic characteristics of internal modules and time delay of signal detection circuit are the major causes of dynamic performance deterioration, which can induce potential system instability in practical control systems. By taking all these factors into consideration, we design a robust control algorithm to realize the high dynamic closedloop detection of IFOG. Finally, experiments show that the improved $0.01 \mathrm{deg} / \mathrm{h}$ high precision IFOG with the proposed control algorithm can achieve fast tracking and good dynamic measurement precision.
\end{abstract}

\section{Introduction}

Interferometer fiber optic gyroscope (IFOG) has become a new and highly stable angular velocity sensor with the development of optical fiber sensing technology $[1,2]$. IFOG is very competitive in the field of inertial device, owing to its advantages over traditional inertial devices, such as no moving parts, simpler structure, higher detection accuracy, and better reliability $[3,4]$.

As digital closed-loop detection scheme becomes the mainstream of current high precision IFOG scheme, optimization of closed-loop control algorithm is of great significance for improving performance in temperature and vibration environment $[5,6]$. In [7], Spammer and Swart analyzed the influence of loop gain on the dynamic characteristic of system and investigated a control algorithm to eliminate steady-state error. Japan Aviation Electronics Industry Limited (JEA) corporation from Japan focused on the winding method of optical fiber coil and closed-loop control technology to improve the temperature and vibration characteristics [8]. An implemented signal processing scheme was proposed to promote the reduction of the source intensity noise around the proper frequency of birefringent IFOG for a better signalto-noise ratio [9]. Thus, the importance of optimization of closed-loop control algorithm is apparent in order to further enhance the system performance.

However, the closed-loop control period of IFOG is in a time scale of several microseconds, while the refresh time of the system output is in a time scale of milliseconds $[10$, 11]. Hence, it is difficult to analyze the working process of IFOG only by the test data of IFOG, especially in the environment of high frequency vibration and shock. By taking the environmental factors into consideration, Litef $\mathrm{GmbH}$ established a simulation system for IFOG and optimized the optical components and design of closed-loop control $[11,12]$. To realize the environmentally insensitive IFOG, Honeywell International proposed a compensating error component by testing the frequency responses of optical path and closed-loop circuit [13]. However, if the inner state variables of closed-loop detection system cannot be monitored in real time, the influence of optical parameters 


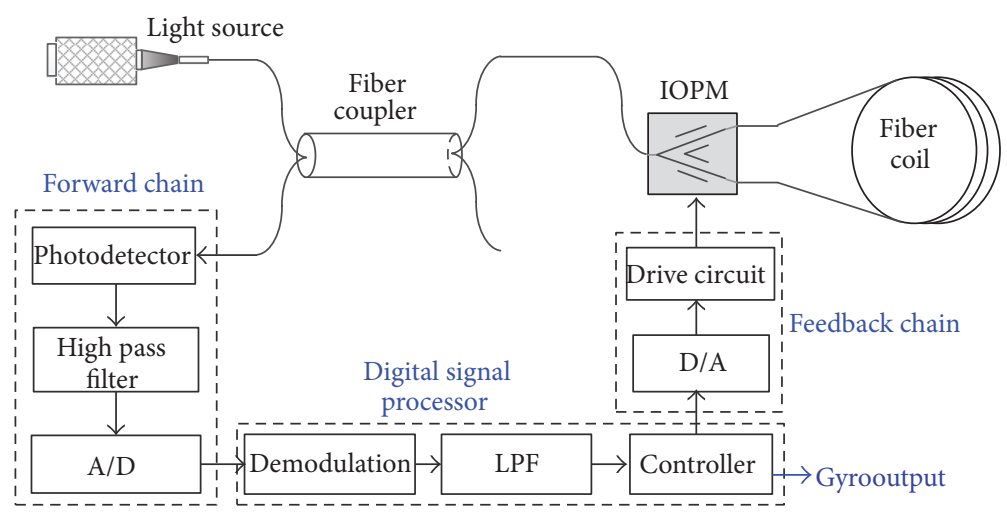

FIGURE 1: The signal detection flowchart of the designed high precision IFOG, which includes a high pass filter to eliminate the DC component in the interference intensity, demodulation, and a low-pass filter (LPF) to extract the modulated nonlinear closed-loop error precisely; a closed-loop controller is needed to obtain the feedback of IFOG and to generate digital phase ramp.

and circuit parameters on the performance of high precision IFOG cannot be analyzed accurately.

In addition, the optical parameters and circuit parameters varying with time and environment can also cause the deterioration of dynamic performance of high precision IFOG. For improving the performance of IFOG, Honeywell International proposed an apparatus for eliminating or reducing vibration-induced errors by applying a variable weighting function at the demodulator or analog-to-digital converter to change the size of the demodulator reference signal or the converter gain, respectively [14]. In respect of the temperature dependency, a precision calibration procedure was described for reducing the resulting errors [15]. In [16], a method based on a radial basis function (RBF) neural network was presented for dynamic angular velocity modeling and error compensation. However, most of the above methods adopted are to compensate error of IFOG according to the built model based on closed-loop output of IFOG. And few works have considered the optical parameters and circuit parameters uncertainty in the dynamic model of the closed-loop system, so that performance of IFOG may not be able to be controlled precisely due to parameter variance with time and environment in engineering practice. With this background, we study the high dynamic model of IFOG with parameters uncertainty and design robust control algorithm to suppress the influence of parameters uncertainty for improving the dynamic characteristics of high precision IFOG.

In this paper, we establish an assistant design platform for high precision IFOG by using the virtual instrument technology, which can monitor the real-time closed-loop state variables and test the dynamic performance of closedloop system. With the assistant design platform, we analyze the influence of optical sensing and detection circuit on the dynamic performance of high precision IFOG. Furthermore, with the consideration of these factors, the high dynamic mathematic model of IFOG with parameters uncertainty is derived and a robust controller is designed to enhance the dynamic measurement performance of high precision IFOG for applications in navigation and aerospace fields.

\section{Analysis Setup of Dynamic Performance of IFOG}

The optical path scheme for high precision IFOG is illustrated in Figure 1. The light from the light source is split by the coupler into two beams, one of which is propagating into integrated optical modulator (IOPM). The light polarized by the IOPM is divided into two light beams that pass through the fiber coil clockwise and anticlockwise, respectively. With the induced Sagnac phase $\Delta \varphi_{s}$, the two beams of light interfere coherently at the IOPM. The time of the light propagating through the fiber coil is $\tau$, which restricts the minimum closed-loop period.

To achieve higher accuracy in angular velocity measurement, we design a signal detection scheme, and the working diagram of the closed-loop detection for IFOG is shown in Figure 1. With the square wave modulation and the phase ramp feedback, the interference intensity can be given by

$$
I=\alpha I_{0}\left\{1+f_{b}(t) \cdot \sin \left[\Delta \varphi_{s}(t)-\Delta \varphi_{f}(t)\right]\right\},
$$

where $\alpha$ is the total loss of the full optical path, $I_{0}$ is the output power of the light source, $\Delta \varphi_{f}(t)$ is the feedback phase stair of the digital phase ramp used to counteract $\Delta \varphi_{s}(t), \Delta \varphi_{s}(t)$ is the Sagnac phase, and $f_{b}(t)$ is the modulating square wave with amplitude \pm 1 and period $2 \tau$. We here introduce a quantity $\Delta \varphi$ that represents the closed-loop error of the IFOG, which is defined by $\Delta \varphi(t)=\Delta \varphi_{s}(t)-\Delta \varphi_{f}(t)$.

To further improve the dynamic measurement precision of IFOG, the influence of optical parameters and signal detection circuits on dynamic performance should be analyzed. However, by the traditional analysis that is only based on the output data of IFOG, the complex closedloop process of IFOG cannot be manifested. And it is also difficult to assess and verify the influence of inner elements' parameters of closed-loop system on the performance of IFOG. In fact, it is difficult to experimentally analyze the dynamic variation of error signal between input and output signals, especially when the system is in high frequency vibration and shock environment. In order to assess the main factors that influence the dynamic characteristics and 


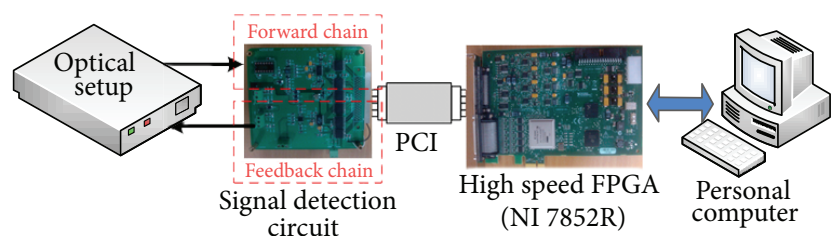

FIGURE 2: The constituent components of the proposed assistant design setup of IFOG, which is composed of four parts: optical setup, signal detection circuit, computer, and NI7852R with an embedded high-speed FPGA.

to optimize the dynamic measurement performance of high precision IFOG, we develop an assistant design setup based on virtual instrument technology as illustrated in Figure 2. The assistant design setup can achieve the high-speed closedloop control with microsecond closed-loop period to detect interference intensity signal and exert the modulating square wave and feedback phase ramp on IOPM. Besides, the proposed detection setup can monitor the output signal of detector and the closed-loop state variables in real time through the digital communication between the embedded high-speed field programmable gate array (FPGA) and the personal computer.

The dynamic performance tests also can be conducted based on this platform. The embedded high-speed FPGA of national instruments (NI) 7852R is utilized to generate a frequency adjustable and phase steerable excitation signal, which is added to the stair ramp to substitute input angular speed in considering the fact that stair ramp imposed on IOPM can introduce phase shift with the same effect as Sagnac phase. We can evaluate the dynamic characteristics by comparing the excitation and output signals since the signal processing unit can generate the excitation signal and process the output data of IFOG simultaneously.

\section{Analysis of Influencing Factors on Dynamic Performance}

3.1. Influence of Optical Characteristics on the Dynamic Performance of High Precision IFOG. The output of photoelectric detector, also served as the input of signal detection unit, is of great importance to the stability and dynamic performance of IFOG. From (1), it is noted that the optical characteristics contain the interference intensity and the physical intrinsic nonlinearity of interference link.

Due to the varying external environment factors, the changing optical parameters result in the fluctuation of interference intensity, such as the unavoidable light power drift with temperature and the attenuation of light power after long-term working. We analyze the relationship between the power variation of light source and the environmental temperature, and the obtained experiment results are shown in Figure 3. Together with the loss of light power in other optical devices, the range of light power variation can reach $\pm 10 \%$ under the condition of temperature varying from $-45^{\circ} \mathrm{C}$ to $+70^{\circ} \mathrm{C}$. As verified by the conducted experiments
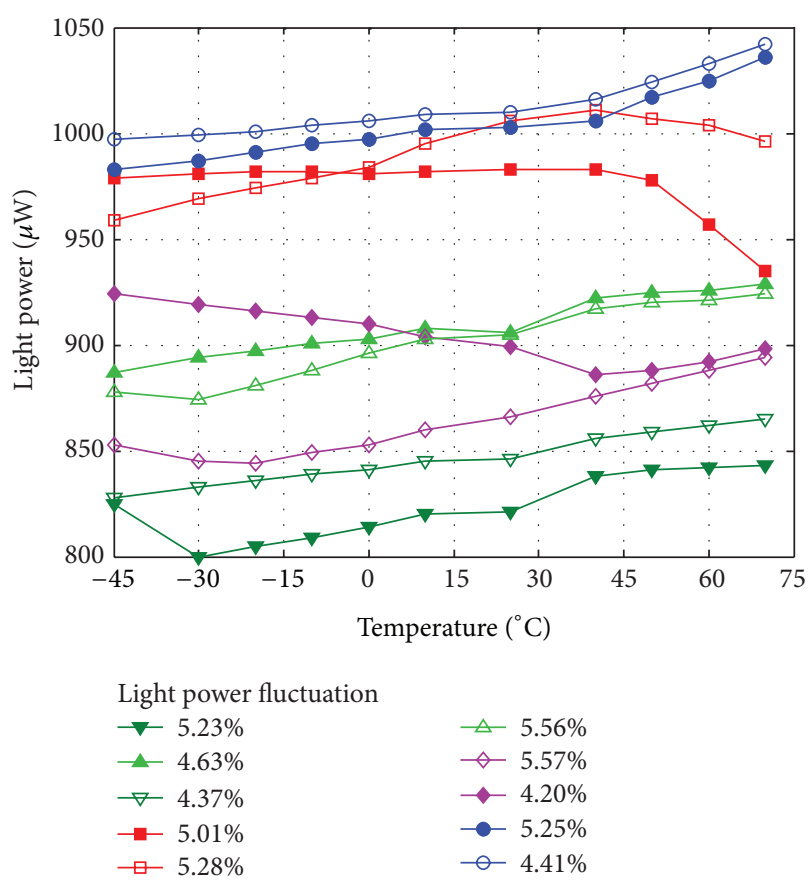

FIGURE 3: The results of experiment tests on the relationship between the light power variation and temperature.

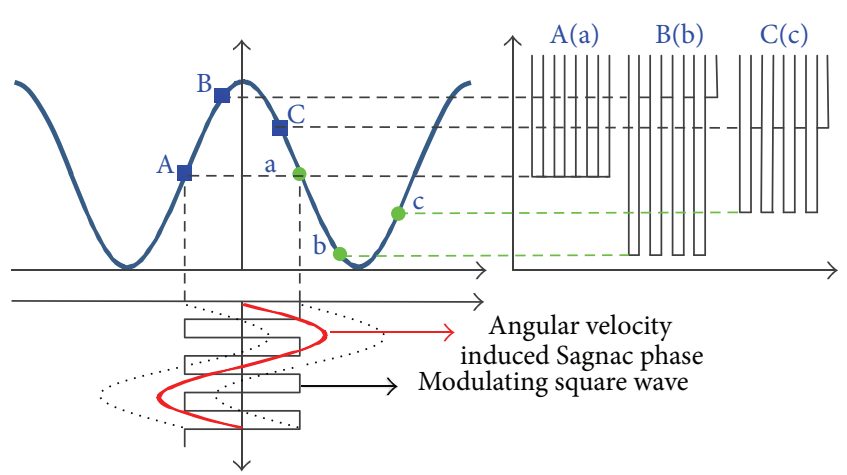

FIGURE 4: The principle of nonlinearity that influences the dynamic performance of the IFOG that adopts the closed-loop scheme of square wave modulation and feedback phase ramp.

based on the assistant design setup, we know that the detection sensitivity of IFOG is not determined by the light power, because the light power is translated into one factor of the gain of forward chain, while the dynamic performance of high precision IFOG is apparently different from the gain variation of forward chain.

The interference link generates a nonlinear sine function, which confines the IFOG's dynamic measurement range. Although the closed-loop scheme of square wave modulation and feedback phase ramp is adopted to suppress the closedloop error near the zero point and to improve the linear characteristic, the IFOG system is a nonlinear system as determined by (1). According to the working principle of our scheme, the linear model used to approximate the nonlinear 


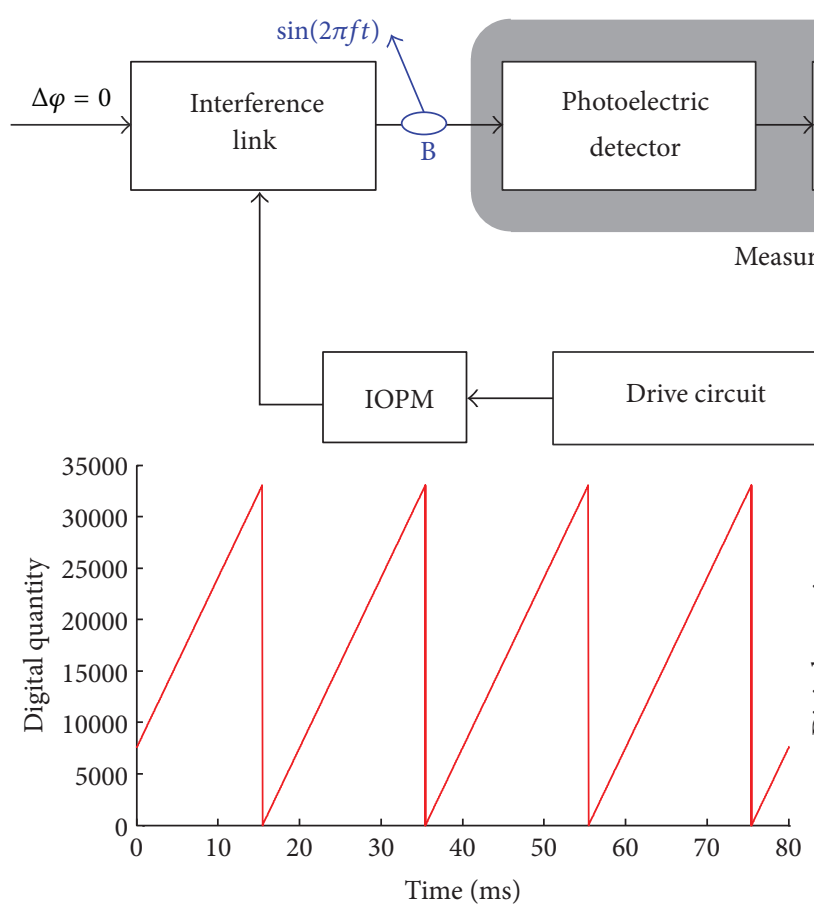

(a)

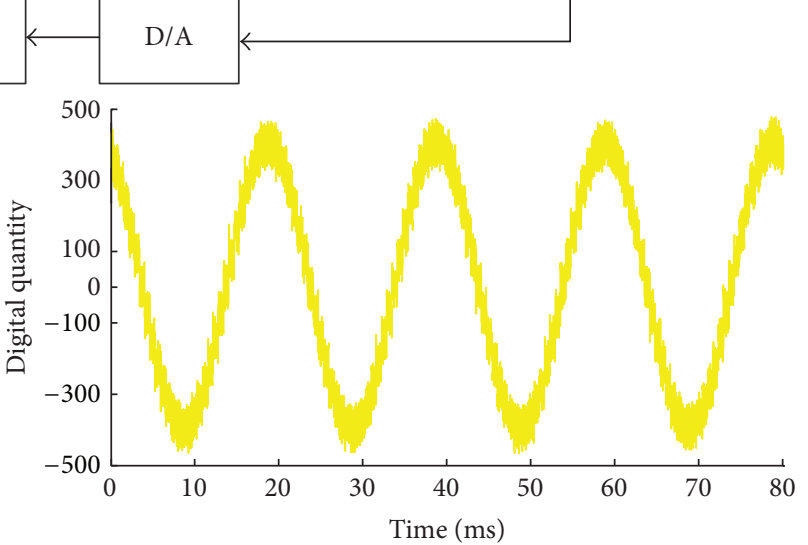

(b)

Figure 5: The testing principle of the frequency characteristic of the whole forward chain; (a) the signal observed at A point; (b) the signal observed at $\mathrm{C}$ point.

system is effective only when the IFOG works near a balance point, such as point $\mathrm{A}$, as denoted in Figure 4 . However, when it works at the state of point $\mathrm{B}$ or point $\mathrm{C}$ due to high frequency or high impulse angular velocity input, the nonlinear characteristic of optical interference link can cause the deterioration of dynamic performance and the potential system instability. In engineering practice, IFOG is not able to precisely measure the high frequency or high impulse angular velocity signal due to the nonlinearity, which might also cause system instability.

3.2. Influence of the Hardware Detection on the Dynamic Performance of the High Precision IFOG. The proposed detection equipment for signal processing can be divided into three parts: the forward chain, the feedback chain, and modulationdemodulation component. To improve the bandwidth of the forward chain and eliminate the DC component of the interference intensity signal, we design a high pass filter in the forward chain. The dynamic characteristics and bandwidth of the forward chain can significantly affect the dynamic performance of the high precision IFOG. However, besides the high pass filter and an A/D converter, the forward chain also includes an optical device-photoelectric detector. It is difficult to analyze the dynamic characteristics of forward chain by using frequency characteristic analyzer.

To solve this problem, we use the assistant design setup to measure the model of the whole forward chain. As illustrated in Figure 5, the assistant design setup is utilized to generate ramp signals at point $\mathrm{A}$. The ramp signal is $u(t)=2 \pi f t$, where $f$ is the frequency of target excitation signal. Then, we make the ingenious application of the nonlinear characteristic of interference link in translating the ramp signal into a sinusoidal signal $\sin (2 \pi f t)$ at point $\mathrm{B}$. The ramp signal has to be reset periodically at the level of $2 \pi$ when it reaches the full scale of DA converter, and the digital signal processor receives the interference signal synchronously at point C. Moreover, the frequency of the sinusoidal signal is controlled by the commercial software Laboratory Virtual Instrument Engineering Workbench (LabVIEW) installed in the computer. Thus the frequency characteristics of the whole forward chain can be calculated based on the assistant design setup. The obtained results demonstrate that the bandwidth of forward chain can reach $10 \mathrm{MHz}$ and satisfy the requirement for high-frequency angular velocity measurement.

To accurately extract the closed-loop error, the output of $\mathrm{A} / \mathrm{D}$ converter is demodulated by multiplying a digital square wave that has the same frequency and phase as that of $f_{b}(t)$, and it also needs a low-pass filter to remove the high frequency components and noise. The response function of the designed low-pass filter is $H(z)=1+\beta_{1} z^{-1}+\beta_{2} z^{-2}+$ $\cdots+\beta_{d} z^{-d}$, where $z^{-1}$ represents the time of one closed-loop period and $\beta_{1}, \ldots, \beta_{d}$ are the coefficients of filter. The higher order of filter can arouse better signal to noise ratio. However, the higher-order items of filter introduce larger time-delay at the same time. The time-delay can intrinsically influence the dynamic performance of the closed-loop system. The feedback chain contains a D/A converter, a D/A drive circuit, 
and the previously mentioned IOPM. A 16 bit D/A converter is adopted and its theoretical bandwidth is up to $50 \mathrm{MHz}$. The $\mathrm{D} / \mathrm{A}$ drive circuit is essentially a translation circuit with bandwidth designed as wide as possible and its practically measured bandwidth is up to $4.547 \mathrm{MHz}$. The IOPM is a conversion circuit that can convert modulation voltage into modulation phase shift and its modulation bandwidth can reach as high as $1 \mathrm{GHz}$. So the feedback chain can be considered as a linear module.

\section{Mathematic Model Establishment and Robust Control Algorithm Design}

The analysis results about the dynamic performance of the optical components and signal detection circuits of high precision IFOG are important for guiding the design of closedloop control algorithm. Prior to the question on how to design an optimized control algorithm, we need to establish the mathematic model of the dynamic performance of closedloop system by taking the influencing factors into consideration, which are obtained from the results of dynamic performance analysis based on the assistant design setup.

Firstly, we analyze the forward chain where the influence of optical parameter uncertainty works. Its bandwidth is much larger than the required bandwidth of high precision IFOG. Therefore the forward chain can be considered as a proportional component with uncertain parameters. The proportional factor of the forward chain, denoted as $k_{1}$, is determined not only by the parameters of detection circuit but also by the interference intensity. We define the variation of proportional factor $k_{1}$ as $\Delta k_{1}$, which reflects the effect of temperature drift on the forward channel. Because the variation range of the gain of the forward channel is bounded, we can denote $\Delta k=H F E$, where $H$ and $E$ are constant matrices with appropriate dimensions describing the parameters' variation of the optical path and detection circuit caused by temperature drift in the forward channel, respectively, and $F$ is an uncertain matrix with appropriate dimension that satisfies $F^{T} F \leq I$. As for the interference intensity $I_{0}$, the gain of high pass filter $k_{H}$, and the gain of $\mathrm{A} / \mathrm{D}$ converter $k_{\mathrm{AD}}$, respectively, we can derive $k_{1}=I_{0}$. $k_{H} \cdot k_{\mathrm{AD}}$. In the feedback chain, due to the limited range of $\mathrm{D} / \mathrm{A}$ converter, the digital phase is reset periodically at the digital level of $2^{n_{\mathrm{DA}}}$ corresponding to $2 \pi$, where $n_{\mathrm{DA}}$ is the conversion bit of the D/A converter. The proportional factor of the feedback chain $k_{m}$ is a constant value satisfying $k_{m}=2 \pi / 2^{n_{\mathrm{DA}}}=K_{\mathrm{D} / \mathrm{A}} \cdot K_{\mathrm{dri}} \cdot K_{\mathrm{IOPM}}$, where $K_{\mathrm{D} / \mathrm{A}}, K_{\mathrm{dri}}$, and $K_{\mathrm{IOPM}}$ are the proportional factors of D/A converter, $\mathrm{D} / \mathrm{A}$ drive circuit, and IOPM, respectively. Then, the feedback phase can be described as $\Delta \varphi_{f}(k)=k_{m} u(k)$, where $u(k)$ is the linear controller described by $u(k)=-k_{m} K_{c} x(k) . K_{c} \in R^{1 \times n}$ is the feedback-gain matrix. The time-delay of the low-pass filter is confined by the closed-loop period, and the nonlinear characteristic of optical interference link deteriorates the high precision IFOG's dynamic performance.

Based on the proposed detection equipment, in consideration of the influence of optical nonlinearity, optical parameter uncertainty, and time-delay of low-pass filter, the mathematic model of high precision IFOG can be described by

$$
\begin{aligned}
x(k+1)= & A x(k)+B\left(k_{1}+\Delta k_{1}\right) \sin \left(-k_{m} K_{c} x(k)\right) \\
& +B\left(k_{1}+\Delta k_{1}\right) \sum_{i=1}^{d} \beta_{i} \sin \left(-k_{m} K_{c} x(k-i)\right),
\end{aligned}
$$

where $x \in R^{n}$ is the state vector with the initial condition $x\left(k_{0}\right)$ for the zero sampling time,

$$
A=\left[\begin{array}{cccc}
1 & 1 & 0 & \cdots \\
0 & 1 & 1 & \cdots \\
\cdots & \cdots & \cdots & \cdots \\
0 & \cdots & 0 & 1
\end{array}\right], \quad B=\left[\begin{array}{c}
0 \\
0 \\
\cdots \\
1
\end{array}\right]
$$

To extract the closed-loop error quickly and optimize the dynamic response characteristic, we assume that the order of low-pass filter is $d=1$.

Now, we investigate the method of designing feedbackgain matrix $K_{c}$ for improving the dynamic performance of system (2) with the initial condition $x\left(k_{0}\right)$. Before the discussion of the main theorem for the design of feedbackgain matrix, some useful definition and lemmas are first elucidated.

Definition 1 (see [17]). System (2) is said to be exponentially stable, if there exist some scalars $\kappa \geq 0$ and $0<\alpha<1$, such that the solution $x(k)$ of system (2) satisfies $\|x(k)\|^{2}<$ $\kappa(1-\alpha)^{k-k_{0}}\left\|x\left(k_{0}\right)\right\|^{2}$, for all $k \geq k_{0}$.

Lemma 2 (see [18] (the Schur complement)). For a given symmetric matrix $S$ with the form $S=\left[S_{i j}\right], S_{11} \in R^{r \times r}$, $S_{12} \in R^{r \times(n-r)}$, and $S_{22} \in R^{(n-r) \times(n-r)}, S<0$ if and only if $S_{11}<0, S_{22}-S_{21} S_{11}^{-1} S_{12}<0$ or $S_{22}<0, S_{11}-S_{12} S_{22}^{-1} S_{21}<0$.

Lemma 3 (see [18]). Suppose that $x \in R^{p}, y \in R^{q}$, and $H$ and $E$ are constant matrices with appropriate dimensions. For any appropriate dimension matrix $F$ satisfying $F^{T} F \leq I$, one has that $2 x^{T} D F E y \leq \varepsilon x^{T} D D^{T} x+(1 / \varepsilon) y^{T} E^{T} E y$ for any $\varepsilon>0$.

In the following discussions, we are interested in analyzing the stability of the IFOG and finding the design rules of the feedback-gain matrix $K_{c}$ to guarantee that the high precision IFOG achieves exponentially dynamic response characteristic.

Theorem 4. System (2) locally solves the exponential stability problem, if there exist positive definite matrices $P, Q, R \in R^{n \times n}$, the feedback-gain matrix $K_{c} \in R^{1 \times n}$, and positive scalars $\alpha, \varepsilon_{1}$, $\varepsilon_{2}$ such that

$$
\psi=\left[\begin{array}{cccc}
\varphi & \varsigma_{1}^{T} R & \varsigma_{2}^{T} R & 0 \\
* & -P & 0 & P H \\
* & * & -R & R H \\
* & * & * & -\varepsilon_{3} I
\end{array}\right]<0
$$


where

$$
\begin{gathered}
\varsigma_{1}=\left[\begin{array}{cccc}
A & 0 & B k_{1} & B k_{1}
\end{array}\right], \\
\varsigma_{2}=\left[\begin{array}{cccc}
A-I & 0 & B k_{1} & B k_{1}
\end{array}\right], \\
\varphi=\left[\begin{array}{cccc}
-(1-\alpha) P+Q-(1-\alpha) R & (1-\alpha) R & \frac{1}{2} \varepsilon_{1} k_{m} K_{c}^{T} & 0 \\
* & -(1-\alpha)(Q+R) & 0 & \frac{1}{2} \varepsilon_{2} k_{m} K_{c}^{T} \\
* & * & -\varepsilon_{1} I+\varepsilon_{3} E^{T} E & \varepsilon_{3} E^{T} E \\
* & * & * & -\varepsilon_{2} I
\end{array}\right] .
\end{gathered}
$$

Proof. See the Appendix.

We use a Lyapunov-based approach to analyze the convergence and the dynamic performance analysis of the high precision IFOG. Theorem 4 provides a sufficient condition to design the control matrix $K_{c}$ for the high precision IFOG with time-delay, nonlinearity, and uncertainty, which guarantees that the system can achieve exponential stabilization. It also should be pointed out that, as deduced from the theoretical analysis on the stability of IFOG, the designed controller can make the dynamic errors of nonlinear IFOG within $(-\pi, \pi)$ exponentially converge to zeros, which are only dependent on the uncertain intensity of light power and the range of time-delay of the low-pass filter. At the same time, the results reveal that the proposed controller has strong robustness, so that the interference intensity is tolerant of temperature shift in a certain range. The theoretical result is of significant importance to the design of good dynamic detection method for the high precision IFOG under complex conditions of engineering application.

\section{Experiment Results}

Experiments are conducted to demonstrate the effectiveness of the previously obtained theoretical results and the dynamic measurement precision of the $0.01 \mathrm{deg} / \mathrm{h}$ IFOG using the optimized control algorithm. In the experiments, the light power is placed in a temperature control chamber where the temperature varies $5^{\circ} \mathrm{C}$ per minute to force the light power changeable in a certain range. We monitor the closedloop state variables in real time to verify the ability of the designed high precision IFOG in suppressing the influence of parameter uncertainty, nonlinearity and time-delay on dynamic performance.

To validate the dynamic response characteristics of IFOG, the experiments are conducted to test the $50 \mathrm{~Hz}$ and $2 \mathrm{kHz}$ frequency response, respectively. The excitation signal is generated by adding digital sinusoidal signal to the feedback phase ramp. We can control the applying time of excitation signal through the detection equipment. The digital signal processor synchronously receives the excitation signal and the output of IFOG so that the precise amplitude attenuation and phase delay can be measured. For the result of $50 \mathrm{~Hz}$ frequency response tests, the deterioration of amplitude is $0.3 \%$ and the phase delay is $0.4^{\circ}$ as shown in Figure 6. In the $2 \mathrm{kHz}$ frequency response tests with the results shown in Figure 7, the relative measurement error is $0.77 \%$ in amplitude and $3^{\circ}$ in phase.

Then, to further validate the dynamic performance of IFOG with the proposed controller, the ramp response of the new high precision IFOG is compared with that of the previous high precision IFOG based on the assistant design setup. The results shown in Figure 8 demonstrate that there exists a dynamic tracking error in the previous high precision IFOG, which explains that the previous scheme of high precision detection reduces the dynamic performance of optical sensing. In contrast, the optimized detection scheme of the high precision IFOG can track the ramp signal rapidly without dynamic tracking error and better tracking performance is achieved as shown in Figure 9.

Furthermore, we conduct the experiments of tracking angular acceleration with $0.01 \mathrm{deg} / \mathrm{h}$ high precision IFOG utilizing the angular rotating platform. Angular rotating platform oscillates at frequency of $28 \mathrm{~Hz}$ so that the output of IFOG forms a sinusoidal trace at various oscillating points. The sinusoidal curve near the zero point can approximate to ramp response, and amplitude of angular acceleration can be controlled by the oscillating amplitude of angular rotating platform. On adopting previous control algorithm and our optimized control algorithm, the results are shown in Figures 10 and 11, respectively. We can see that the experiment results based on the angular rotating platform agree well with the results utilizing the assistant design setup, both of which verify that the optimized robust control algorithm can track the angular acceleration better than the previous control algorithm. All the above experiments demonstrate that the high precision IFOG system with our optimized control algorithm can effectively suppress the influence of parameter uncertainty, nonlinearity, and time-delay, so that a 


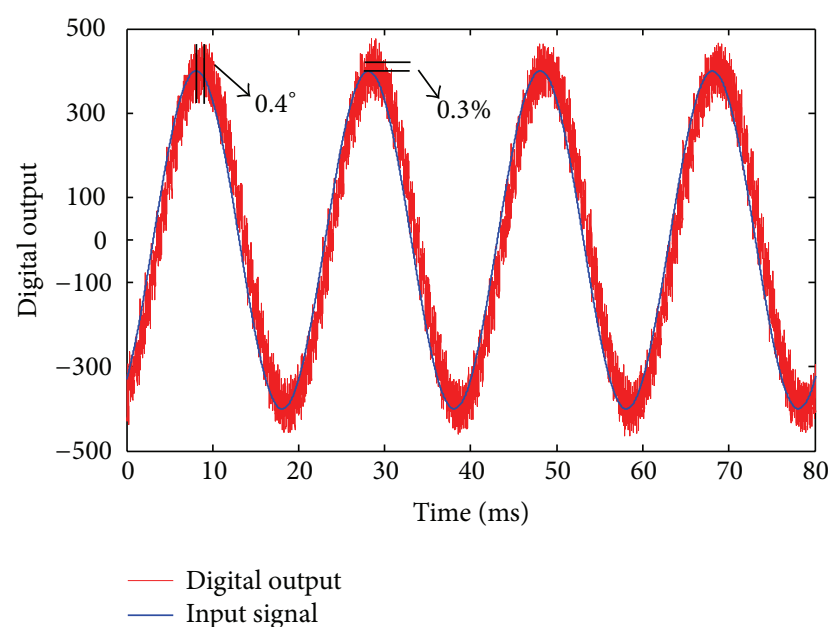

FIgURE 6: The result of the $50 \mathrm{~Hz}$ frequency response test of the optimized IFOG.

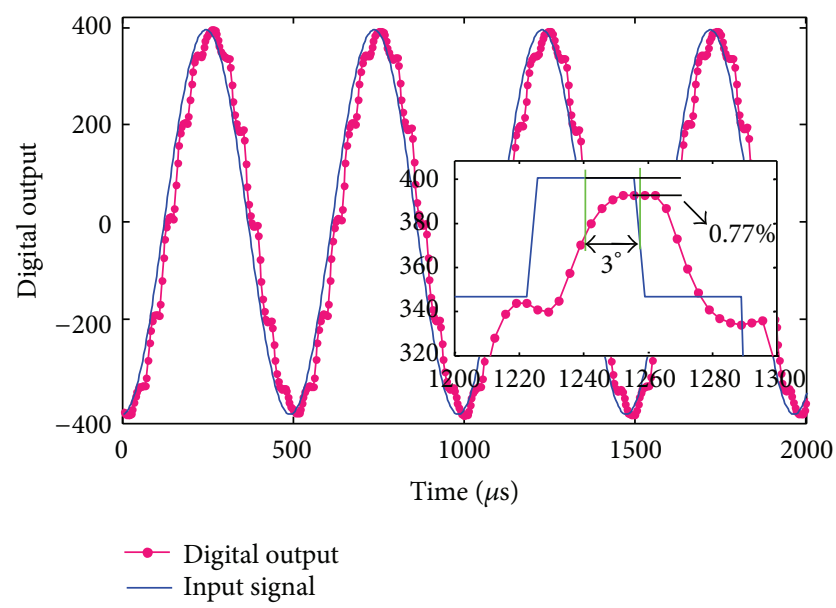

FIGURE 7: The result of the $2 \mathrm{kHz}$ frequency response test of the optimized IFOG.

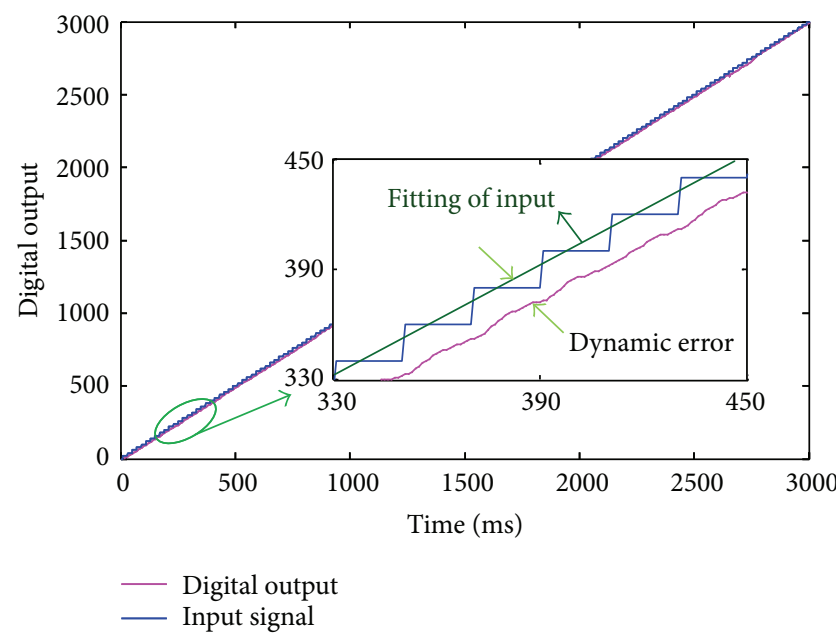

FIGURE 8: The result of the ramp response test of the previous $0.01 \mathrm{deg} / \mathrm{h}$ IFOG.

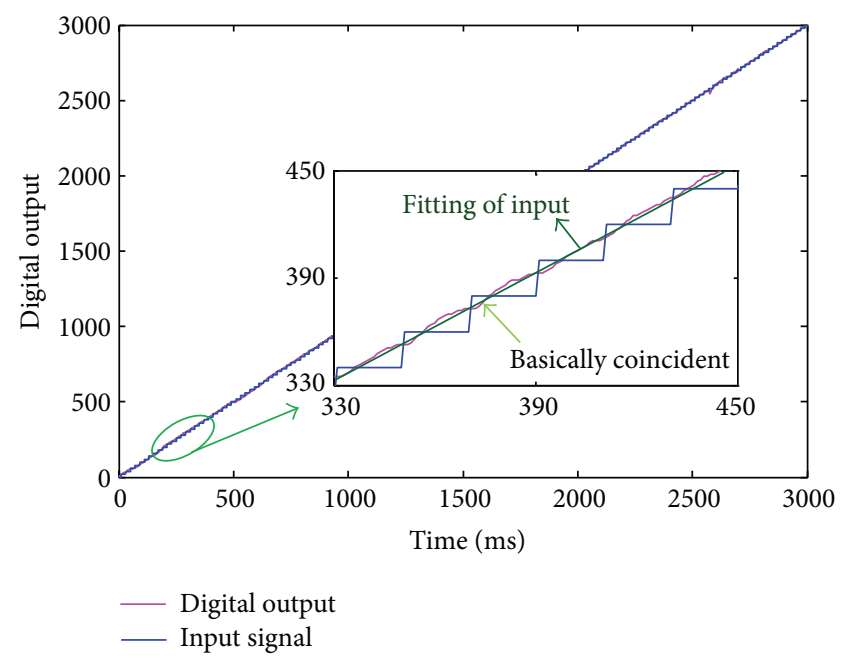

FIGURE 9: The result of the ramp response test of the optimized $0.01 \mathrm{deg} / \mathrm{h}$ IFOG.

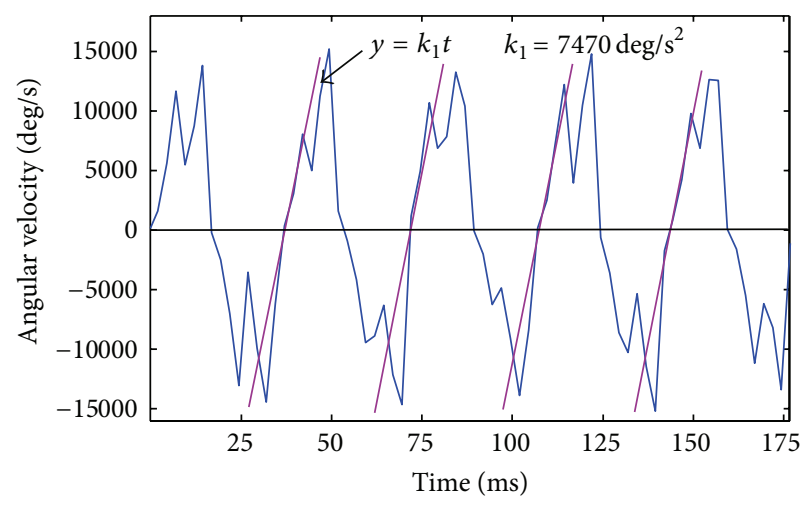

FIGURE 10: The output of $0.01 \mathrm{deg} / \mathrm{h}$ IFOG adopting previous control algorithm when the angular acceleration is $7470 \mathrm{deg} / \mathrm{s}^{2}$.

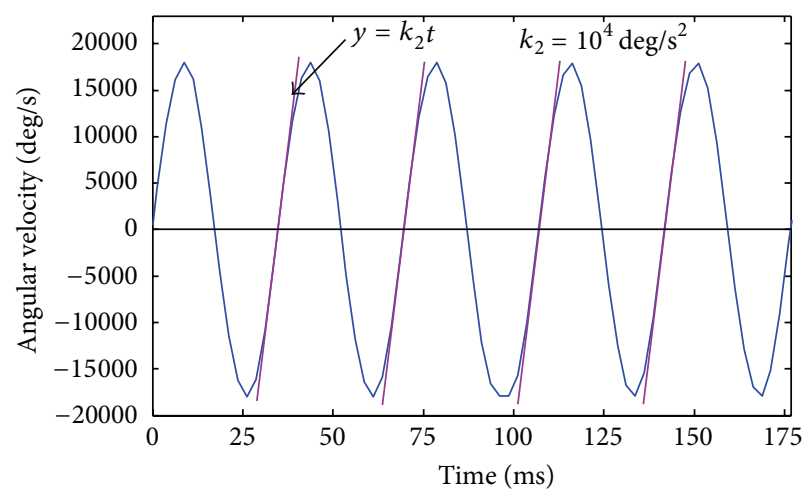

FIGURE 11: The output of $0.01 \mathrm{deg} / \mathrm{h}$ IFOG adopting our optimized control algorithm, when the angular acceleration is $10000 \mathrm{deg} / \mathrm{s}^{2}$.

better dynamic measurement performance is achieved. These strategies could be applied in the engineering systems of high precision IFOGs to improve their dynamic measurement precision meeting the demand of inertial navigation systems. 


\section{Conclusion}

In engineering practice, the closed-loop detection scheme can improve the measurement precision of IFOG. In this work, the influence of optical sensing and closed-loop signal detection on the dynamic performance of high precision IFOG is analyzed based on the established assistant design platform. We find that the several important facts that restrict the high dynamic property of IFOG include that (1) the interference link introduces the nonlinearity, (2) the optical parameter variation causes the light power fluctuation, and (3) the time of the light propagating through the fiber coil limits the closed-loop period and the time-delay of the lowpass filter. By taking these influencing factors into consideration, we propose a robust control algorithm to improve the dynamic characteristics of high precision IFOG. The obtained experiment results demonstrate the effectiveness of the proposed closed-loop design method. The improved dynamic measurement precision is of great significance in expanding the scope of application of IFOG.

\section{Appendix}

Proof of Theorem 4. We consider the main influencing factors to obtain the exponential stability condition of the high precision IFOG system. The following Lyapunov function is chosen for system (2):

$$
\begin{aligned}
V(k)= & x^{T}(k) P x(k)+\sum_{s=k-d}^{k-1} x^{T}(s)(1-\alpha)^{k-s-1} Q x(s) \\
& +d \sum_{s=-d}^{-1} \sum_{m=k+s}^{b-1} \eta^{T}(k)(1-\alpha)^{k-m-1} R \eta(k),
\end{aligned}
$$

where $\eta(k)=x(k+1)-x(k)$ and $P, Q$, and $R$ are defined as those in Theorem 4.

Calculating $\Delta V(k)=V(k+1)-V(k)$ along the solution of system (2), we have that

$$
\begin{aligned}
\Delta V(k) & +\alpha \Delta V(k) \\
= & x^{T}(k+1) P x(k+1)-(1-\alpha) x^{T}(k) P x(k)
\end{aligned}
$$

$$
\begin{aligned}
& +x^{T}(k) \mathrm{Q} x(k)-(1-\alpha)^{d} x^{T}(k-d) Q x(k-d) \\
& +d^{2} \eta^{T}(k) R \eta(k)-d(1-\alpha)^{d} \sum_{m=k-d}^{k-1} \eta^{T}(m) R \eta(m) .
\end{aligned}
$$

As $d=1$ in the digital filter of the high precision IFOG, we have

$$
\begin{aligned}
& -d \sum_{m=k-d}^{k-1} \eta^{T}(m) R \eta(m) \\
& \quad=-(x(k)-x(k-1))^{T} R(x(k)-x(k-1)) .
\end{aligned}
$$

We find that $\sin (x)$ satisfies the Lipschitz condition and is a monotonically ascending function for $x \in(-\pi, \pi)$. That is, there exists a function $M(k)$, such that $\sin \left(\Delta \varphi_{f}(k)\right)=$ $M(k) \Delta \varphi_{f}(k)$ for $\Delta \varphi_{f}(k) \in(-\pi, \pi)$, where $\|M(k)\| \leq l_{f}$ with $l_{f}=1$. Then, the following inequality holds:

$$
\sin \left(\Delta \varphi_{f}(k)\right)^{T}\left(\sin \left(\Delta \varphi_{f}(k)\right)-l_{f} \Delta \varphi_{f}(k)\right) \leq 0,
$$

where $\Delta \varphi_{f}(k) \in(-\pi, \pi)$.

For any two scalars $\varepsilon_{1}>0$ and $\varepsilon_{2}>0$, we have that

$$
\begin{aligned}
& \Delta V(k)+\alpha \Delta V(k) \\
& \leq \Delta V(k)+\alpha \Delta V(k) \\
& \quad-\varepsilon_{1} \sin \left(\Delta \varphi_{f}(k)\right)^{T}\left(\sin \left(\Delta \varphi_{f}(k)\right)-l_{f} \Delta \varphi_{f}(k)\right) \\
& \quad-\varepsilon_{2} \sin \left(\Delta \varphi_{f}(k-1)\right)^{T}\left(\sin \left(\Delta \varphi_{f}(k-1)\right)\right. \\
& \left.\quad-l_{f} \Delta \varphi_{f}(k-1)\right) \\
& \leq \xi^{T}(k)\left(\widetilde{\varphi}+\widetilde{\varsigma}_{1}^{T} P \widetilde{\varsigma}_{1}+\widetilde{\varsigma}_{2}^{T} P \widetilde{\varsigma}_{2}\right) \xi(k),
\end{aligned}
$$

where

$$
\begin{gathered}
\widetilde{\varsigma}_{1}=\left[\begin{array}{llll}
A & 0 & B\left(k+\Delta k_{1}\right) & B\left(k+\Delta k_{1}\right)
\end{array}\right], \\
\widetilde{\varsigma}_{2}=\left[\begin{array}{llll}
A-I & 0 & B\left(k+\Delta k_{1}\right) & B\left(k+\Delta k_{1}\right)
\end{array}\right], \\
\xi(k)=\left[\begin{array}{llll}
x^{T}(k) & x^{T}(k-1) & \sin ^{T}\left(\Delta \varphi_{f}(k)\right) & \sin ^{T}\left(\Delta \varphi_{f}(k-1)\right)
\end{array}\right]^{T}, \\
\widetilde{\varphi}=\left[\begin{array}{cccc}
-(1-\alpha) P+Q-(1-\alpha) R & (1-\alpha) R & \frac{1}{2} \varepsilon_{1} k_{m} K_{c}^{T} & 0 \\
* & -(1-\alpha)(Q+R) & 0 & \frac{1}{2} \varepsilon_{2} k_{m} K_{c}^{T} \\
* & * & -\varepsilon_{1} I & 0 \\
* & * & * & -\varepsilon_{2} I
\end{array}\right] .
\end{gathered}
$$


By Lemma 2, we can see that $\widetilde{\varphi}+\widetilde{\varsigma}_{1}^{T} P \widetilde{\varsigma}_{1}+\widetilde{\varsigma}_{2}^{T} P \widetilde{\varsigma}_{2}<0$, if and only if

$$
\left[\begin{array}{ccc}
\widetilde{\varphi} & \widetilde{\varsigma}_{1}^{T} P & \widetilde{\varsigma}_{2}^{T} R \\
* & -P & 0 \\
* & * & -R
\end{array}\right]<0
$$

Because $\Delta k=H F E,(\mathrm{~A} .7)$ can be rewritten as

$$
\left[\begin{array}{ccc}
\widetilde{\varphi} & \varsigma_{1}^{T} P & \varsigma_{2}^{T} R \\
* & -P & 0 \\
* & * & -R
\end{array}\right]+M^{T} F(k) N+N^{T} F^{T}(k) M<0,
$$

where $\varsigma_{1}$ and $\varsigma_{2}$ are defined as that in Theorem $4, N=$ $\left[\begin{array}{llllll}0 & 0 & E & E & 0 & 0\end{array}\right]$, and $M=\left[\begin{array}{llllll}0 & 0 & 0 & 0 & H^{T} P & H^{T} R\end{array}\right]$.

By Lemma 3, (A.8) holds if and only if there exists a positive constant $\varepsilon_{3}$ such that

$$
\left[\begin{array}{ccc}
\widetilde{\varphi} & \varsigma_{1}^{T} P & \varsigma_{2}^{T} R \\
* & -P & 0 \\
* & * & -Q
\end{array}\right]+\varepsilon_{3}^{-1} M^{T} M+\varepsilon_{3} N^{T} N<0 .
$$

Thus a sufficient condition for $\Delta V(k)+\alpha V(k)<0$ is equivalent to $\psi<0$ by the Schur complement lemma. If $\Delta V(k)+\alpha V(k)<0$, we have that $V(k) \leq(1-\alpha)^{k-k_{0}} V\left(k_{0}\right)$ along any trajectory of system (2). Then, IFOG can obtain the exponential stability.

Furthermore, we have that

$$
\begin{aligned}
\lambda_{1} x^{T}(k) x(k) & \leq V(k) \\
& \leq(1-\alpha)^{k-k_{0}} V(k) \\
& \leq \lambda_{2}(1-\alpha)^{k-k_{0}} x^{T}\left(k_{0}\right) x\left(k_{0}\right),
\end{aligned}
$$

where $\lambda_{1}=\min \lambda(P)$ is the minimum eigenvalue of $P, \lambda_{2}=$ $\max \lambda(P)+d \max \lambda(Q)+2 d^{2}(d+1) \max \lambda(R)$, and $\max \lambda(\cdot)$ is the maximum eigenvalue of the matrix.

That means that $\|x(k)\| \leq \sqrt{\lambda_{1} / \lambda_{2}}(1-\alpha)^{k-k_{0}}\left\|x\left(k_{0}\right)\right\|$ by (A.10) and we can conclude that system (2) is locally and exponentially stable. This completes the proof.

\section{Acknowledgments}

This work was supported by the National Aeronautics Foundation of China (no. 20100851016) and the National Natural Science Foundation of China (no. 61101007).

\section{References}

[1] G. M. Williams and E. J. Friebele, "Space radiation effects on erbium-doped fiber devices: sources, amplifiers, and passive measurements," IEEE Transactions on Nuclear Science, vol. 45, no. 3, pp. 1531-1536, 1998.

[2] J. Jin, H. Tian, N. Song, and C. Zhang, "Stellar sensor assistant fiber optic gyroscope drift extraction," Transactions of the Japan Society for Aeronautical and Space Sciences, vol. 53, no. 179, pp. 40-46, 2010.

[3] H. C. Lefevre, The Fiber-Optic Gyroscope, Artech House, London, UK, 1993.
[4] N. Barbour and G. Schmidt, "Inertial sensor technology trends," IEEE Sensors Journal, vol. 1, no. 4, pp. 332-339, 2001.

[5] R. Zhu, Y. Zhang, and Q. Bao, "A novel intelligent strategy for improving measurement precision of FOG," IEEE Transactions on Instrumentation and Measurement, vol. 49, no. 6, pp. 1183$1188,2000$.

[6] R. Azor, I. Y. Bar-Itzhack, J. K. Deutschmann, and R. R. Harman, "Angular-rate estimation using delayed quaternion measurements," Journal of Guidance, Control, and Dynamics, vol. 24, no. 3, pp. 436-443, 2001.

[7] S. J. Spammer and P. L. Swart, "Open-loop fibre optic gyroscope with wide dynamic range and source variation insensitivity," in Proceedings of the 15th Anniversary Conference on SPIE Fiber Optic Gyros, vol. 1585, pp. 215-225, 1992.

[8] A. Ohno, S. Motohara, S. Usul et al., "Development of fiberoptic-gyroscope with environmental ruggedness," in Proceedings of the 15th Anniversary Conference on SPIE Fiber Optic Gyros, pp. 82-88, 1992.

[9] R. C. Rabelo, R. T. de Carvalho, and J. Blake, "SNR enhancement of intensity noise-limited FOGs," Journal of Lightwave Technology, vol. 18, no. 12, pp. 2146-2150, 2000.

[10] J. Jin and S. Lin, "Parameters optimization of interferometric fiber optic gyroscope for improvement of random walk coefficient degradation in space radiation environment," Optics and Lasers in Engineering, vol. 50, pp. 1106-1112, 2013.

[11] J. Kunz, M. W. Kemmier, G. Spahlinger et al., "Design of an ASIC for a commercial small volume fiber optic gyro," in Proceedings of the 20th Anniversary Conference on SPIE Fiber Optic Gyros, vol. 2837, pp. 98-105, 1996.

[12] G. Spahlinger, M. Kemniler, M. Ruf et al., "Error compensation via signal correlation in high precision closed-loop fiber optic gyros," in Proceedings of the 20th Anniversary Conference on SPIE Fiber Optic Gyros, vol. 2837, pp. 218-227, 1996.

[13] S. J. Sanders, J. E. Lewis, S. Mosor et al., "Systems and methods for environmentally insensitive high-performance fiber-optic gyroscopes," US 2013/0044328 A1.

[14] G. A. Sanders, R. C. Dankwort, and R. A. Bergh, "Vibration rectification error reducer for fiber optic gyroscope," US 5,946,097.

[15] H. Chung, L. Ojeda, and J. Borenstein, "Sensor fusion for mobile robot dead-reckoning with a precision-calibrated fiber optic gyroscope," in Proceedings of the IEEE International Conference on Robotics and Automation (ICRA '01), pp. 3588-3593, May 2001.

[16] Y.-S. Zhang, Y. Wang, T. Yang, R. Yin, and J. Fang, "Dynamic angular velocity modeling and error compensation of onefiber fiber optic gyroscope (OFFOG) in the whole temperature range," Measurement Science and Technology, vol. 23, no. 2, Article ID 025101, 2012.

[17] D. Zhang, L. Yu, and W. A. Zhang, "Exponential $H_{\infty}$ filtering for a class of switched stochastic hybrid systems with mixed time delays and random missing measurements," Asian Journal of Control, vol. 14, no. 3, pp. 807-816, 2013.

[18] S. Boyd, L. El Ghaoui, E. Feron, and V. Balakrishnan, Linear Matrix Inequalities in System and Control Theory, vol. 15, Society for Industrial and Applied Mathematics (SIAM), Philadelphia, Pa, USA, 1994. 


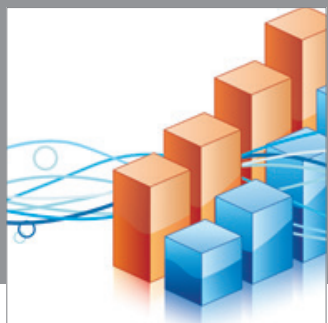

Advances in

Operations Research

mansans

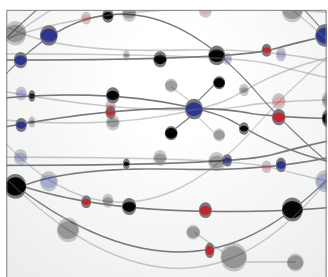

The Scientific World Journal
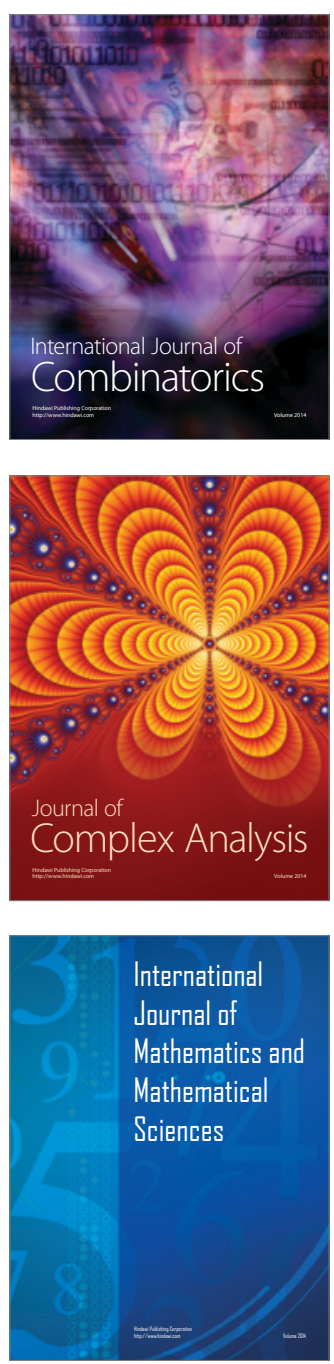
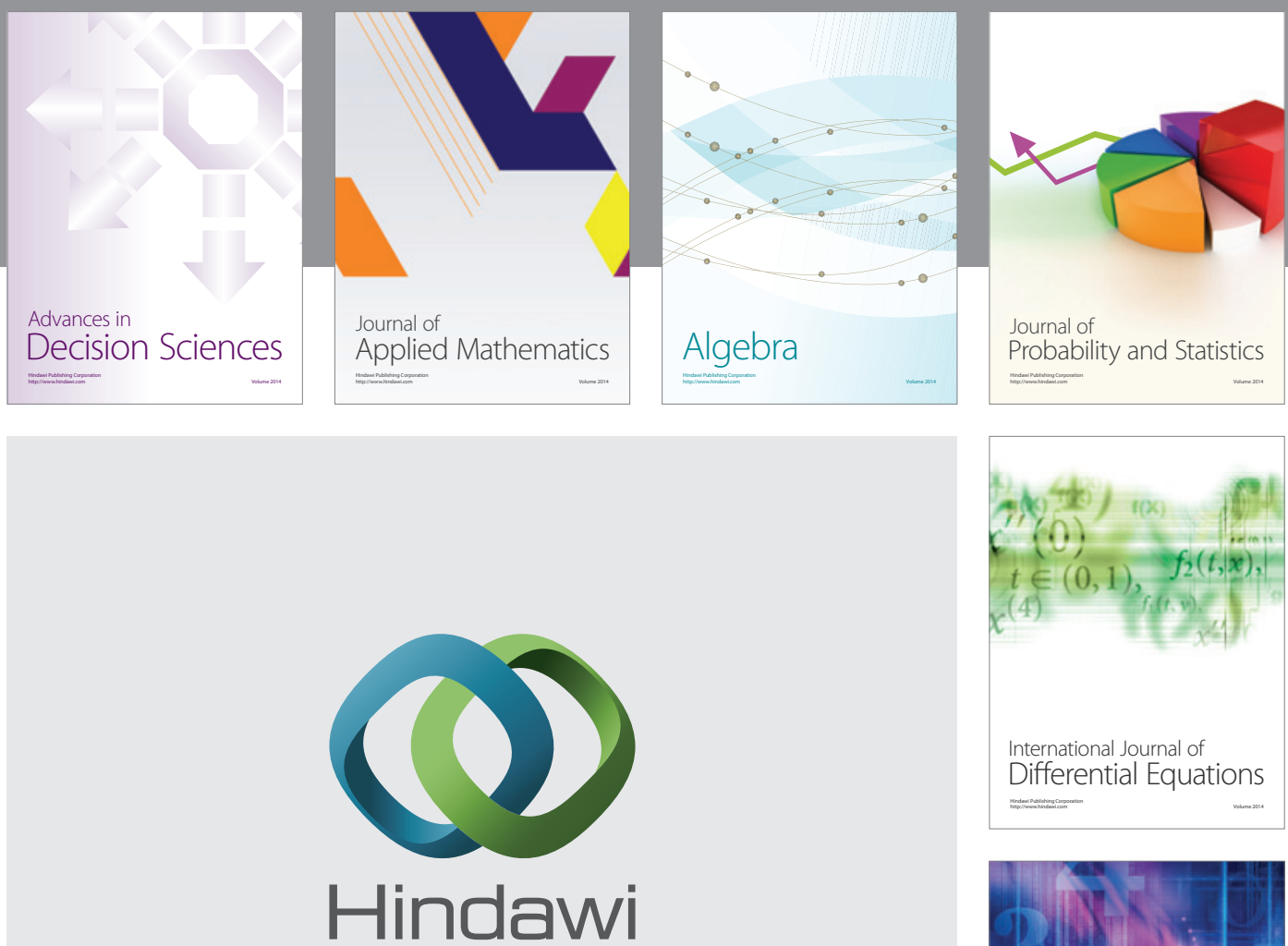

Submit your manuscripts at http://www.hindawi.com
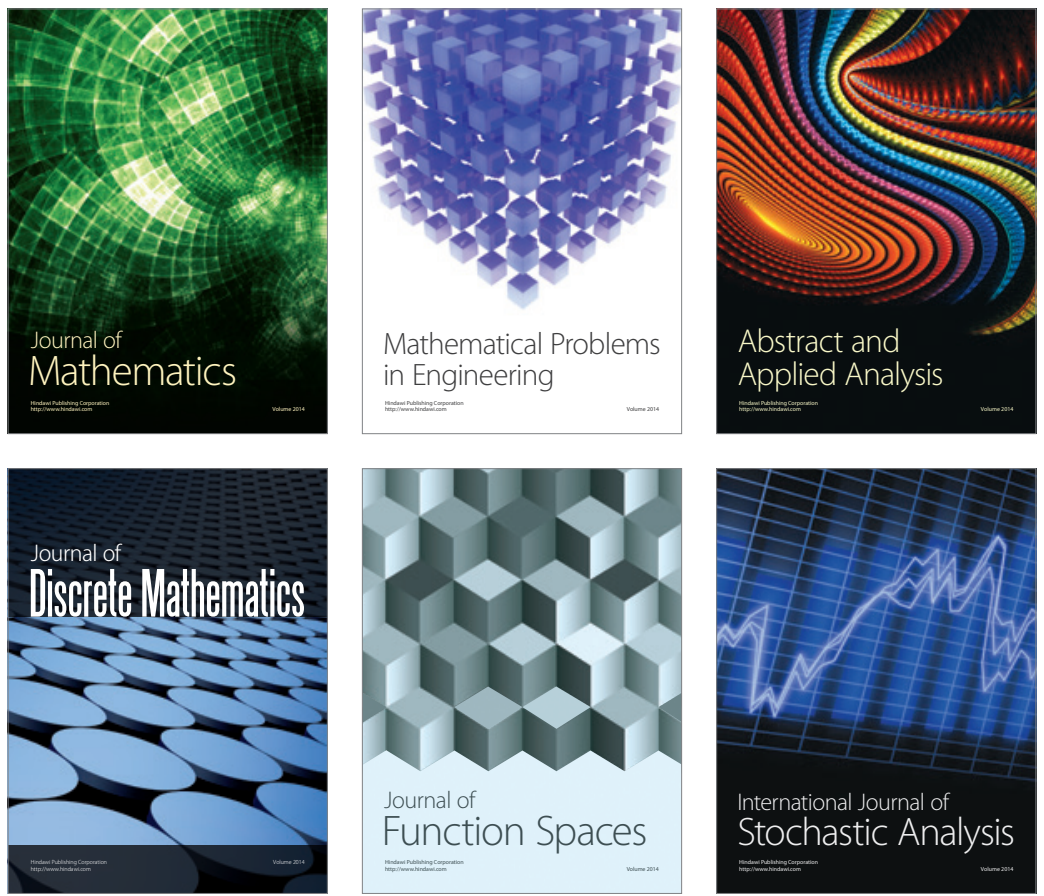

Journal of

Function Spaces

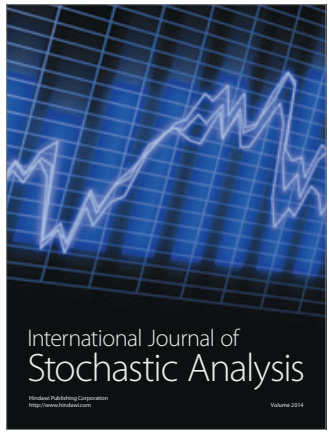

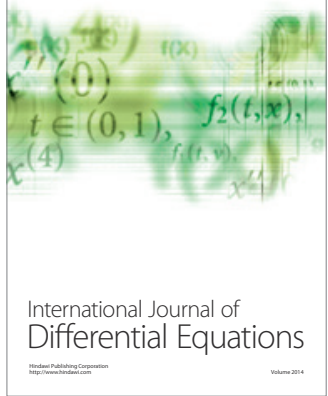
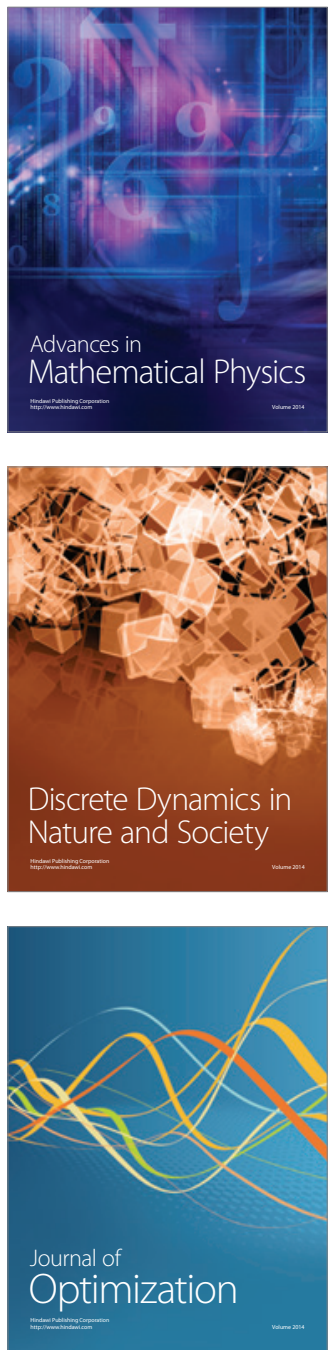